\title{
NEUROSCIENCES ET PSYCHIATRIE
}

\author{
J.Ph. BOULENGER*
}

Le récent Colloque national des Neurosciences de Bordeaux rassemblait la plupart des chercheurs français travaillant dans le domaine, actuellement en pleine expansion, de la neurobiologie. Cependant, $\grave{a}$ côté de l'accumulation des travaux fondamentaux qui, chaque année, font progresser de façon spectaculaire nos connaissances sur le système nerveux central, comment ne pas être frappé par l'absence relative d'applications cliniques notables de près de vingt années de recherches continues dans le domaine de la psychiatrie biologique. Il est en effet facile de constater que, depuis les années 60, aucun des index biologiques étudiés ne s'est avéré susceptible d'apporter en clinique des renseignements d'ordre diagnostique ou pronostique fiables et que, mis à part le lithium, aucun médicament plus efficace que les molécules qui existaient à cette époque n'est apparu sur le marché, malgré la multiplication des produits psychotropes mis à la disposition du corps médical. Ce contraste frappant entre les progrès de la biologie expérimentale et l'absence de débouchés pratiques de ces travaux suscite deux ordres de commentaires, les uns généraux et touchant à la méthodologie des recherches psychiatriques, les autres portant plus précisément sur l'organisation de la recherche clinique dans notre pays.

Constatant cette impasse relative de la psychiatrie biologique, certains ont, à juste titre, remis en question les stratégies de recherche utilisées jusque là dans ce domaine et notamment l'adéquation des concepts cliniques classiques aux tentatives de corrélations avec l'activité de systèmes neurobiologiques divers. En même temps que se dessine un vaste mouvement de reconceptualisation nosographique (initié par le DSM III), se développent donc des recherches visant à définir la pathologie psychiatrique en fonction de dimensions cliniques ou cognitives nouvelles plus aisément utilisables dans le cadre d'études biologiques. Ce retour à un abord plus phénoménologique de la pathologie psychiatrique ne nie donc pas l'intérêt potentiel de la neurobiologie, mais vise au contraire à mieux utiliser dans le futur les progrès des neurosciences : méconnaitre cette évolution de la recherche clinique serait une source potentielle de conflits entre biologistes et cliniciens et certaines interventions au Colloque de Bordeaux ont d'ailleurs été révélatrices de l'incompréhension qui pourrait en résulter.

Aussi important peut-être est le problème des moyens mis à la disposition des cliniciens dans un domaine de recherche où la biologie possède une position prévalente. Là encore, un effort de compréhension s'impose afin de déterminer ce qui peut distinguer les besoins de la recherche clinique par rapport à ceux de la recherche expérimentale. Besoin d'unités de recherche clinique tout d'abord, les créations nouvelles, en nombre très limité, remplaçant à peine celles fermées au cours de ces dernières années. Besoin de moyens spécifiques également car, contrairement aux études fondamentales, les études cliniques s'avèrent beaucoup plus longues à réaliser, nécessitent des efforts considérables pour le recrutement de patients adaptés et génèrent des données multiples. Outre leur lenteur, les recherches cliniques se caractérisent donc par leur besoin en personnel qualifié; la création de postes d'infirmiers ou d'assistants de recherche serait certainement un élément décisif pour permettre aux équipes existantes d'atteindre le rendement scientifique qu'on peut attendre d'elles.

*INSERM-Centre Esquirol - CHU Côte de Nacre - 14033 CAEN Cedex, France 
Enfin, la position des chercheurs cliniciens plein temps pose de délicats problèmes d'intégration au sein d'un système hospitalo-universitaire qui méconnait parfois les exigences méthodologiques de la recherche et doit consacrer la majorité de son temps aux autres tâches qui lui sont dévolues. Dans ce sens, la création de groupes autonomes bénéficiant de structures et d'un recrutement propres (type Institut) devrait être favorisée, à l'exemple de la plupart des autres pays occidentaux. De telles structures devraient bien sûr assurer des soins et participer à l'enseignement, mais leur objectif unique devrait être la recherche clinique, une discipline qui, comme la recherche expérimentale, ne saurait être développée dans le cadre d'un temps partiel. Intermédiaires entre des organismes de recherche où prédominent les fondamentalistes et un système hospitalo-universitaire qui tend parfois à considérer leur activité comme marginale, les psychiatres-chercheurs ne peuvent que difficilement trouver les conditions d'une activité optimale. La méconnaissance des problèmes spécifiqués de la recherche en psy. chiatrie et ses tendances actuelles ne peut que renforcer les sentiments d'inefficacité qui se font jour parmi eux. Espérons que les efforts entrepris permettront d'atteindre et de dépasser le seuil critique d'organisation et de moyens au-delà duquel la recherche clinique pourra démontrer son efficacité et son utilité dans des domaines qui ne seront pas forcément ceux de la biologie ; l'attitude consistant à penser que tout est déjà en place pour atteindre ce but ne peut que promouvoir l'incompréhension qui se fait jour entre ces deux approches, pourtant complémentaires, de la recherche psychiatrique.

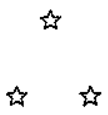

